\title{
FAMILIAL CASES OF DOWN'S SYNDROME A PSU DIC (21) (q22) AND A ROB (14q21q) IN COUSINS
}

\author{
Takako Matsubara, ${ }^{1,2}$ Kazuso Iinuma,,${ }^{2, *}$ Yasuo Nakagome, ${ }^{1}$ \\ and Tsunehiro YoKOCHI ${ }^{2}$ \\ ${ }^{1}$ Department of Human Genetics, National Institute of Genetics, \\ Mishima, Shizuoka 411, Japan \\ ${ }^{1}$ Shizuoka Children's Hospital, Shizuoka, Shizuoka, 420 Japan
}

\begin{abstract}
Summary A patient is described of a girl aged 6 months with typical features of Down's syndrome. Chromosomal studies revealed a 46,XX, $-21,+$ psu $\operatorname{dic}(21) \quad($ pter $\rightarrow$ cen $\rightarrow \mathrm{q} 22:: \mathrm{q} 22 \rightarrow$ pter $)$ karyotype. A maternal cousin also had Down's syndrome. His karyotype was $46, \mathrm{XY},-14$, $+\operatorname{rob}(14 \mathrm{q} 21 \mathrm{q})$.
\end{abstract}

\section{INTRODUCTION}

Familial occurrence of Down's syndrome is rather rare except for those caused by either translocation or mosaicism of one of the parents. Familial aggregation of trisomy 21 or trisomy 21 plus another case due to de novo translocation, have been described (Priest et al., 1963; Zellweger, 1968). A sib with two cases of Down's syndrome due to $21 \mathrm{q} 21 \mathrm{q}$ "de novo" translocation was also described (Schmidt and Nitowsky, 1977).

In the present communication, a family with two cases of Down's syndrome is described. Chromosome studies revealed two different structural rearrangements, a psu $\operatorname{dic}(21)$ and a rob(14q21q), both being de novo.

\section{CASE REPORT}

The proband (M.K.) was a Japanese female. She was the first child of the mother and the father aged 27 and 24 years, respectively, at the time of her birth. The parents were not consanguineous and phenotypically normal. Family history was negative except for a maternal cousin with Down's syndrome (Fig. 1).

The proband was born at term after an uneventful gestation. The birth weight was $2,730 \mathrm{~g}$ and the length $47 \mathrm{~cm}$. Physical examinations at 4 months of age

Received January 23, 1981

* Present address: Ebina Kosei Hospital, Ebina, Kanagawa 243, Japan. 


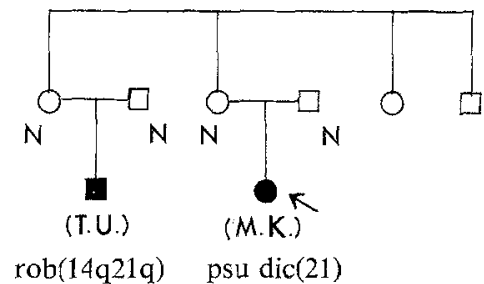

Fig. 1. Family tree.

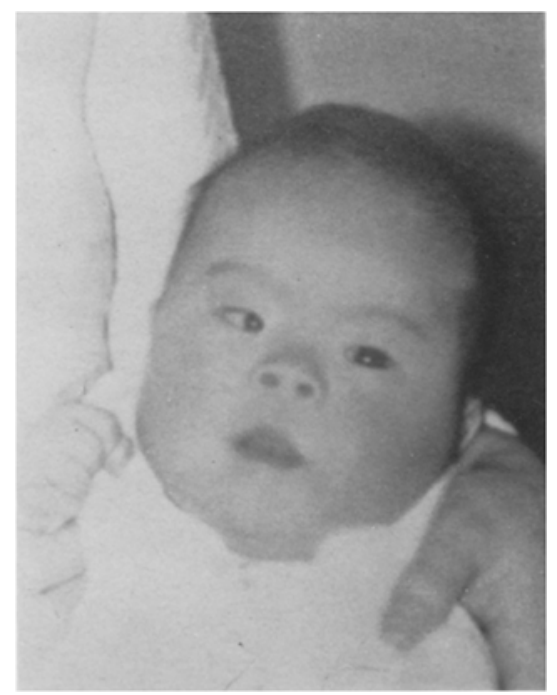

Fig. 2. Patient (M,K.) at 4 months of age.

revealed various features of Down's syndrome; growth retardation, upward slanting of palpebral fissures, strabismus, hypertelorism, bilateral epicanthic folds, wide and flattened nasal bridge and general hypotonia (Fig. 2). The mouth was small and kept open most of the time. There was systolic heart murmur on the left sternal border in the 4th intercostal space. The ECG, X-ray films of the chest and ultrasonic cardiography were also suggestive of the presence of congenital heart disease. Caridiac catheterization, however, was not possible because of her sudden death. No simian line was observed on palms. Hallucal patterns were distal loop (r) and tibial loop (1). She died abruptly at 6 months of age after episodes of dyspnea and cyanosis. Autopsy was not granted.

The maternal cousin (T.U.) was the only child of healthy, noncousanguineous parents. The mother and father were 25 and 34 years old at the time of the delivery. $\mathrm{He}$ was born at term and weight $2,950 \mathrm{~g}$. The crown-heel length and head circumference were $45.5 \mathrm{~cm}$ and $33.8 \mathrm{~cm}$, respectively. At birth obstetricians made a diagnosis of Down's syndrome based on clinical findings. It was later confirmed by various 


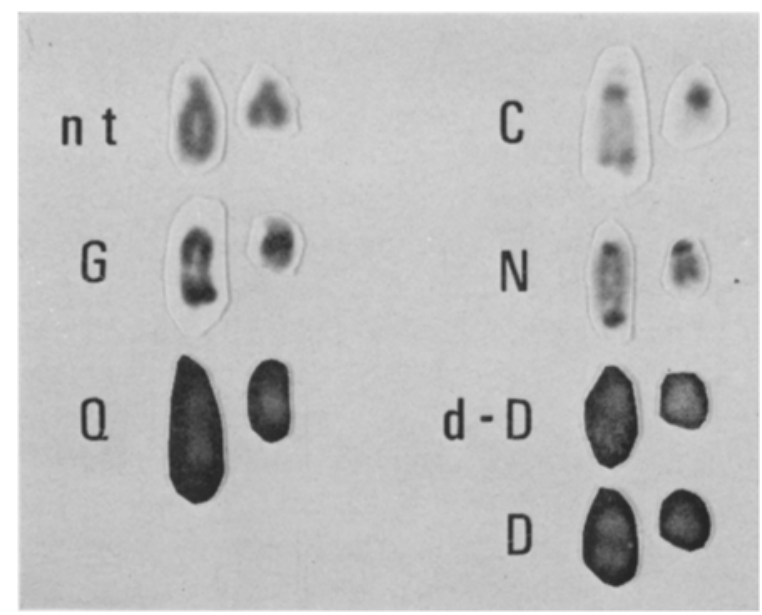

Fig. 3. Chromosome No. 21 pair of the proband. nt, no treatment [Giemsa staining]; G, GTG; Q, QFQ; C, CBG; N, NOR [Ag-I method]; d-D, distamycin-DAPI; D, DAPI.

physical findings (e.g., typical facial appearance, general muscle hypotonia and diastasis recti), X-ray examinations (e.g., markedly reduced acetabular and iliac angle) and dermatoglyphic findings obtained by 21 months of age. The latter included $R$ on both right 5 th and left 4 th fingers, $\mathrm{U}$ on 7 finger tips and bilateral high axial triradii $\left(t^{\prime \prime}\right)$.

\section{CYTOGENETIC STUDIES}

Proband (M.K.): The standard leukocyte culture revealed a 46,XX,Gq + karyotype in every cells examined. The $\mathrm{Gq}+$ chromosome had satellites on both of its ends and either of them were involved in a satellite association. Both C-and Nbanding (Sumner et al., 1972; Bloom and Goodpasture, 1976) revealed positive material near the end of the long arm in addition to the centromere (the short arm for the latter). Both $\mathrm{G}$ and $\mathrm{Q}$ staining revealed that the $\mathrm{Gq}+$ chromosome consisted of two no. 21 chromosomes fused together at near the ends of the long arm, points of breakage being at band 21q22 (Fig. 3). The Gq + chromosome represented, in fact, a dicentric chromosome with one of its centromeres inactivated, the karyotype being 46,XX,-21,+psu dic(21) (pter $\rightarrow$ cen $\rightarrow$ q $22::$ q22 $\rightarrow$ pter). Distamycin-DAPI staining revealed a large and bright segment on only one of the two ends. Thus the dicentric chromosome was formed through translocation (tdic) rather than isodicentric (idic) rearrangement. Both parents showed normal karyotype. Only the mother's cells were available for distamycin-DAPI staining and neither of no. 21 pair had an enlarged and bright short arm.

The maternal cousin (T.U.) had a $46, X Y,-14,+(14 q 21 q)$ complement. Both parents showed a normal karyotype. 


\section{DISCUSSION}

When there is either familial translocation or mosaicism involving a $21(\mathrm{~s})$, multiple cases of Down's syndrome are sometimes observed within a sib. On the other hand, it is very unusual to see, if at all, the same caused by de novo formation of different types of structural rearrangement in a family.

In the present communication, familial cases of Down's syndrome is described. The proband was a typical example of Down's syndrome based on clinical findings, however, his karyotype was $46, \mathrm{XX}, \mathrm{Gq}+$. Banding studies revealed that the abnormal chromosome was, in fact, dicentric with one of its two centromeres inactivated, i.e., psu $\operatorname{dic}(21)$ (pter $\rightarrow$ cen $\rightarrow \mathrm{q} 22:: \mathrm{q} 22 \rightarrow$ pter). This particular chromosome was presumably formed during the process of gametogenesis in one of the parents as both of them showed a normal karyotype by the analysis of somatic cells.

One of the maternal cousin was also affected by Down's syndrome. His karyotype was $46, \mathrm{XY},-14,+\operatorname{rob}(14 \mathrm{q} 21 \mathrm{q})$. This rearrangement, again, was arisen de novo from normal parents. Whether there are any common causes between the two de novo rearrangements is not known. It is quite possible that their occurrence is fortuitous.

It has been established that in a dicentric chromosome, one of two centromeres are inactivated, and further, only the active one is positively stained by the Cd staining (Nakagome et al., 1976; Maraschio et al., 1980). In the present case, the $\operatorname{dic}(21)$ chromosome assumed a monocentric appearance and only the active centromere was $\mathrm{Cd}$ positive. The other was presumably inactivated and became $\mathrm{Cd}$ negative. The details of Cd-band study will be described elsewhere.

The present case is trisomic for no. 21 except for a distal part of q22 band $(q 22 \rightarrow q$ ter) and showed typical features of Down's syndrome in agreement with the literature. According to Hagemeijer et al. (1977), the triplication of the $21 \mathrm{q} 22$ band is essential for the phenotype of Down's syndrome. On the other hand, the triplication of the most distal end of it may not be necessary (Poissonnier et al., 1976; and Raoul et al., 1976).

Acknowledgements This study was supported in part by Grant-in-Aid for Scientific Research Results, No. 357650 from the Ministry of Education, Science and Culture of Japan, and by a grant from the Ministry of Health and Welfare, Japan.

\section{REFERENCES}

Bloom, S.E., and Goodpasture, C. 1976. An improved technique for selective silver staining of nucleolar organizer regions in human chromosomes. Hum. Genet. 34: 199-206.

Hagemeijer, A., and Smit, E.M.E. 1977. Partial trisomy 21. Further evidence that trisomy of band $21 \mathrm{q} 22$ is essential for Down's phenotype. Hum. Genet. 38: 15-23.

Maraschio, P., Zuffardi, O., and Curto, F.L. 1980. Cd bands and centrometric function in dicentric chromosomes. Hum. Genet. 54: 265-267.

Jpn. I. Human Genet. 
Nakagome, Y., Teramura, F., Kataoka, K., and Hosono, F. 1976. Mental retardation, malformation syndrome and partial $7 \mathrm{p}$ monosomy $[45, \mathrm{XX}, \mathrm{tdic}(7 ; 15)(\mathrm{p} 21 ; \mathrm{p} 11)]$. Clin. Genet. 9: 621624.

Poissonnier, M., Saint-Paul, B., Dutrillaux, B., Chassaigne, M., Gruyer, P., and de BlignieresStrouk, G. 1976. Partial trisomy (21q21 $\rightarrow 21 \mathrm{q} 22.2)$. Ann. Génét. 19: 69-73.

Priest, J.H., Bryant, J., and Motulsky, A.G. 1963. Down's syndrome: Trisomy and a de-novo translocation in a family. Lancet $2: 411-412$.

Raoul, O., Carpentier, S., Dutrillaux, B., Mallet, R., and Lejeune, J. 1976. Trisomies partielles du chromosome 21 par translocation maternelle t(15;21) (q262; q21). Ann. Génét. 19: 187-190.

Schmidt, R., and Nitowsky, H.M. 1977. Recurrence of apparent de novo 21/21 translocation trisomy in a sibship. J. Pediat. 90: 841-842.

Sumner, A.T. 1972. A simple technique for demonstrating centromeric heterochromatin. Exp. Cell Res. 75: 304-306.

Zellweger, H. 1968. Familial aggregation of the 21-trisomy syndrome. Ann. N.Y. Acad. Sci.155: 784-792.

Vol. 26, No. 1, 1981 